\title{
Phonon-induced scattering of excited electrons and holes on (110) noble metal surfaces
}

\author{
S. V. Eremeev, ${ }^{1,2,3}$ S. S. Tsirkin, ${ }^{2}$ and E. V. Chulkov ${ }^{3,4, *}$ \\ ${ }^{1}$ Institute of Strength Physics and Materials Science, pr. Academicheskii 2/4, 634021 Tomsk, Russia \\ ${ }^{2}$ Tomsk State University, pr. Lenina 36, 634050 Tomsk, Russia \\ ${ }^{3}$ Donostia International Physics Center (DIPC), Paseo Manuel de Lardizabal 4, San Sebastián/Donostia, 20018 Basque Country, Spain \\ ${ }^{4}$ Departamento de Física de Materiales and Centro de Física de Materiales (CFM) (CSIC-UPV/EHU), Facultad de Ciencias \\ Químicas, Universidad del Pais Vasco/Euskal Herriko Unibertsitatea, Apdo. 1072, San Sebastián/Donostia, 20080 Basque Country, Spain
}

(Received 15 March 2010; revised manuscript received 31 May 2010; published 16 July 2010)

\begin{abstract}
A detailed theoretical study of electron-phonon interaction in occupied and unoccupied $\bar{Y}$ surface states on $\mathrm{Cu}(110), \mathrm{Ag}(110)$, and $\mathrm{Pd}(110)$ is carried out. We show that in the unoccupied surface state on $\mathrm{Cu}(110)$ and $\operatorname{Ag}(110)$ the $e$-ph coupling parameter $\lambda$ is about three times smaller than that in the occupied surface state due to distinct phonon modes that contribute to $e$-ph scattering. The difference in mechanisms of phonon-mediated scattering for excited holes and electrons results from distinct spatial localization of occupied and unoccupied states. In spite of both $\bar{Y}$ surface states on $\operatorname{Pd}(110)$ are unoccupied they have spatial distribution similar to that on other considered noble metal surfaces that leads to different phonon-mediated scattering in these states too.
\end{abstract}

DOI: 10.1103/PhysRevB.82.035424

PACS number(s): 73.20.-r, 68.35.Ja, 72.10.Di

\section{INTRODUCTION}

Electronic surface states (SSs) play a crucial role in electronic and atomic processes on metal surfaces. SSs affect work function of metals, ${ }^{1}$ increase the electron density in the surface layers, ${ }^{2}$ thus influencing the relaxation of surface layers ${ }^{3,4}$ and the energy shift of core electrons. ${ }^{5,6}$ SSs are important for electron and hole dynamics on metal surfaces $^{7,8}$ and for epitaxial metal-on-metal growth processes. ${ }^{9,10}$ Electronic excitations in SSs also play an important role in photodissociation and photodesorption processes as well as in catalytic reactions. ${ }^{11,12}$ Of particular interest are the (110) surfaces of fcc metals which show an intrinsic structural anisotropy. It is well known that more open crystal surfaces such as (110) of fcc metals or stepped surfaces are generally more catalytically active than closepacked surfaces. ${ }^{13}$ For example, $\operatorname{Pd}(110)$ is ten times more active than $\operatorname{Pd}(111)$ for hydrogenation. ${ }^{14}$

The linewidth $\Gamma$ of a SS is formed by three decay processes: electron-electron scattering $\left(\Gamma_{e-e}\right)$, electron-phonon interaction $\left(\Gamma_{e-p h}\right)$, and electron scattering by defects $\left(\Gamma_{e-d}\right)$. Thermal broadening of SSs originates primarily from the linear temperature dependence of the electron-phonon $(e-p h)$ coupling term. ${ }^{15,16}$ Additional thermal broadening is from temperature-dependent part of $\Gamma_{e-d}$, which depends on thermal defect concentration. ${ }^{17,18}$ At zero-temperature limit the $e$-ph contribution to $\Gamma$ is normally of a few millielectron volt, however, at room temperature (RT) it becomes one of the most important contributions.

During the last decade, the $e-p h$ interaction in surface structures has been a subject of intensive theoretical and experimental investigations. Most of these investigations have been performed for SSs formed on close-packed metal surfaces ${ }^{15,16,19-27}$ while only few papers have been devoted to the study of $e-p h$ coupling in SS formed on the (110) noble metal surfaces. ${ }^{28,29}$

Unlike (111) and (001) surfaces of noble metals, where the energy gap at the $\bar{\Gamma}$ point is determined by the projection of bulk states in $\Gamma L$ and $\Gamma X$ directions, respectively, on the
(110) surface of these metals the energy gap is formed at the Brillouin zone (BZ) boundary, at the $\overline{\mathrm{Y}}$ point, by the projection of bulk states along the direction which does not cross the bulk BZ center. ${ }^{30,31}$ Two surface states are present in this gap: the occupied $\left(p_{y}\right.$ like) and the unoccupied $\left(s-p_{z}\right.$ like $)$ surface states. On $\mathrm{Cu}(110)$ the occupied surface state lies at $-0.51 \mathrm{eV}$ below the Fermi energy $\left(E_{\mathrm{F}}\right)($ Ref. 29) and the unoccupied one has energy of $1.7 \mathrm{eV}^{32} \mathrm{On} \mathrm{Ag}(110)$ the occupied SS has an energy of $-0.106 \mathrm{eV}$ (Ref. 33) while the unoccupied SS lies at $1.6 \pm 0.2 \mathrm{eV}$ above $E_{\mathrm{F}} \cdot{ }^{34}$ The band structure of $\operatorname{Pd}(110)$ is different from that of the (110) surfaces of copper and silver: the energy gap at the $\bar{Y}$ point is situated above the Fermi level and both surface states are unoccupied.

The early estimation of $\Gamma_{e-p h}$ for the occupied SS on $\mathrm{Cu}(110)$ had been done in temperature-dependent photoemission experiment. ${ }^{29}$ From the linear relationship between the photoemission peak width and temperature the $e$-ph coupling parameter $\lambda=0.23 \pm 0.02$ was obtained. The estimation of $\Gamma_{e-p h}$ for SSs on $\operatorname{Ag}(110)$ was performed by Dounce et al. ${ }^{28}$ by using temperature-dependent resonantly enhanced second-harmonic generation (SHG) spectroscopy. They estimated the $d \Gamma / d T$ slope as $0.072 \pm 0.014 \mathrm{meV} / \mathrm{K}$, which corresponds to $\lambda=0.13 \pm 0.02$. It is important to note that $\Gamma_{e-p h}$ as well as total surface-state transition linewidth obtained from the SHG experiments includes contributions from both surface states, and the obtained $\lambda$ is an average over occupied and unoccupied states. These experimental estimations $^{28,29}$ of the $e-p h$ coupling have not been supported yet by the theoretical analysis.

In this paper we present the results of a detailed theoretical study of the electron-phonon interaction of excited holes and electrons in occupied and unoccupied surface states on the (110) surface of noble metals $(\mathrm{Cu}, \mathrm{Ag}$, and $\mathrm{Pd})$. We do not include the $\mathrm{Au}(110)$ surface into consideration since, in contrast to $\mathrm{Cu}, \mathrm{Ag}$, and $\mathrm{Pd}$, it is reconstructed. We show that the $e$-ph coupling parameter $\lambda$ in the unoccupied $\overline{\mathrm{Y}}$ surface state on the (110) considered noble metal surface is about three times smaller than that in the occupied surface state due 
to distinct phonon modes providing $e$-ph scattering. We will mainly focus on $\mathrm{Cu}(110)$ because of similarity of the scattering mechanism on the other (110) metal surfaces.

\section{COMPUTATIONAL METHOD}

The phonon-induced lifetime broadening of a surface band state with momentum $\mathbf{k}_{i}$ and binding energy $\epsilon_{\mathbf{k}_{i}}, \Gamma_{e-p h}$, is given by ${ }^{35}$

$$
\begin{aligned}
\Gamma_{e-p h}\left(\mathbf{k}_{i}\right)= & 2 \pi \int_{0}^{\omega_{\max }}\left\{\alpha^{2} F_{\mathbf{k}_{i}}^{E}(\omega)\left[1+n(\omega)-f\left(\epsilon_{\mathbf{k}_{i}}-\omega\right)\right]\right. \\
& \left.+\alpha^{2} F_{\mathbf{k}_{i}}^{A}(\omega)\left[n(\omega)+f\left(\epsilon_{\mathbf{k}_{i}}+\omega\right)\right]\right\} d \omega,
\end{aligned}
$$

where $n(\omega)$ and $f(\epsilon)$ are Bose and Fermi distribution functions, respectively, $\alpha^{2} F_{\mathbf{k}_{i}}^{E}(\omega)$ and $\alpha^{2} F_{\mathbf{k}_{i}}^{A}(\omega)$ are Eliashberg spectral functions that take into account both phonon emission $(E)$ and phonon absorption $(A)$ processes.

The strength of the $e$-ph coupling in one-electron state $\left(\Psi_{\mathbf{k}_{i}}, \epsilon_{\mathbf{k}, i}\right)$ described by parameter $\lambda_{\mathbf{k}, i}$ is defined as the first reciprocal moment of the Eliashberg spectral function $\alpha^{2} F_{\mathbf{k}, i}(\omega)($ Ref. 35)

$$
\lambda_{\mathbf{k}, i}=2 \int_{0}^{\omega_{\max }} \frac{\alpha^{2} F_{\mathbf{k}, i}(\omega)}{\omega} d \omega .
$$

The spectral function in quasielastic scattering approximation $\delta\left(\epsilon_{\mathbf{k}_{i}}-\epsilon_{\mathbf{k}_{f}} \pm \omega_{\mathbf{q}, \nu}\right) \approx \delta\left(\epsilon_{\mathbf{k}_{i}}-\epsilon_{\mathbf{k}_{f}}\right)$ is given by

$$
\begin{aligned}
\alpha^{2} F_{\mathbf{k}, i}(\omega)= & \frac{1}{(2 \pi)^{2}} \int d^{2} \mathbf{q} \sum_{\nu, f} \delta\left(\omega-\omega_{\mathbf{q}, \nu}\right) \\
& \times\left|g\left(\mathbf{k}_{i}, \mathbf{k}_{f}, \mathbf{q}, \nu\right)\right|^{2} \delta\left(\epsilon_{\mathbf{k}, i}-\epsilon_{\mathbf{k}, f}\right),
\end{aligned}
$$

where $g\left(\mathbf{k}_{i}, \mathbf{k}_{f}, \mathbf{q}, \nu\right)$ - the $e$-ph matrix element which reflects the probability of electron scattering from the initial state $\Psi_{\mathbf{k}_{i}}$ with energy $\epsilon_{\mathbf{k}, i}$ to the final state $\Psi_{\mathbf{k}_{f}}$ with energy $\epsilon_{\mathbf{k}, f}$ by the phonon $\omega_{\mathbf{q}, v}$. The sum in Eq. (3) is carried out over all final electron states $\Psi_{\mathbf{k}_{f}}$ and all possible phonon modes. The $e$ - $p h$ matrix element is

$$
g\left(\mathbf{k}_{i}, \mathbf{k}_{f}, \mathbf{q}, \nu\right)=\left(\frac{1}{2 M \omega_{\mathbf{q}, \nu}}\right)^{1 / 2} \times\left\langle\Psi_{\mathbf{k}_{i}}\left|\hat{\varepsilon}_{\mathbf{q}, \nu} \cdot \nabla_{\mathbf{R}} V_{s c}\right| \Psi_{\mathbf{k}_{f}}\right\rangle,
$$

where $M$ is the atomic mass, $\hat{\varepsilon}_{\mathbf{q}, \nu}$ are the phonon polarization vectors, and $\nabla_{\mathbf{R}} V_{s c}$ is the gradient of the screened oneelectron potential with respect to atom displacements from their equilibrium positions $\mathbf{R}$.

In the present calculations we use an approach similar to that used earlier for studying $e$-ph interaction in surface and quantum-well states on the close-packed surfaces. ${ }^{18,21,24,36,37}$ This approach combines three independent approximations to evaluate the $e$-ph coupling matrix elements [Eq. (4)].

(1) One-electron potential $V_{s c}$ is represented by the Ashcroft $^{\text {pseudopotential }}{ }^{38}$ screened within Thomas-Fermi approximation. We used experimentally derived core radius in the Ashcroft pseudopotential for $\mathrm{Cu}$ and $\mathrm{Ag}$ and fit it for palladium to reproduce $a b$ initio calculated ${ }^{27} \lambda$ for the $\bar{\Gamma}$ surface state on $\operatorname{Pd}(111)$.
(2) To calculate phonon spectrum we used embedded atom method interatomic potentials. To simulate a semiinfinite (110) fcc metal surface a thin-film model of a twodimensional (2D) periodic slab consisting of 33 atomic layers is used. The same slab thickness we use to calculate electronic band structure. The chosen thickness prevents interaction between two opposite surfaces of the film. The calculations of vibrational spectra are carried out by the dynamical matrix method. Diagonalization of the matrix gives the eigenfrequencies and the polarization vectors of vibrations. The local vibration densities of states $F_{X, Y, Z}(\omega)$ were obtained by projecting these eigenmodes onto the surface atoms in a given $(X, Y$, and $Z)$ direction.

(3) To describe electronic band structure of the (110) surface we used a 2D model proposed recently. ${ }^{30,31}$ Unlike (001) and (111) surfaces, which can be described by a pseudopotential varying in the direction perpendicular to the surface only, ${ }^{39}$ the description of band structure of the (110) surface requires a two-variable-dependent pseudopotential. This pseudopotential is given by ${ }^{30,31}$

$$
V(y, z)=V^{(0)}(z)+V^{(1)}(z) \cos \left(\frac{2 \pi}{a} y\right),
$$

where $a$ is the lattice parameter. In this model the wave functions are written as

$$
\Psi_{\mathbf{k}}(\mathbf{r})=\sqrt{\frac{1}{\Omega_{0} N_{\|}}} e^{i k_{x} x} \sum_{g_{y}} e^{i\left(k_{y}+g_{y}\right) y} \phi_{k_{y}}^{g_{y}}(z),
$$

where $\Omega_{0}$ is the square of the $2 \mathrm{D}$ unit cell, $N_{\|}$is number of atoms in the layer, and $g_{y}=\frac{2 \pi}{a} n$ ( $n$ is integer). The fitting of the pseudopotential parameters for the (110) surface of noble metals was performed in Refs. 30 and 31. To eliminate the effect of finite slab thickness (discreteness of the bulk electronic bands) we employ sufficiently large $\left(1 \times 10^{-2} \mathrm{Ha}\right)$ Gaussian broadening parameter in $\delta(\epsilon)$ smearing in evaluations of the spectral function [Eq. (3)].

The $e$-ph matrix element within this model can be written as

$$
\begin{aligned}
g\left(\mathbf{k}_{i}, \mathbf{k}_{f}, \mathbf{q}, \nu\right)= & \left(\frac{1}{2 M \omega_{\mathbf{q}, \nu} \Omega_{0}}\right)^{1 / 2} \sum_{g_{y}^{i}, g_{y}^{f}} \int d z \phi_{i, k_{y}^{i}}^{g_{y}^{i}}(z) \\
& \times G_{\nu}\left(\mathbf{q}, \mathbf{G}_{\|}, g_{y}^{f}-g_{y}^{i}, z\right) \phi_{f, k_{y}^{f}}^{g_{y}^{f}}(z) .
\end{aligned}
$$

Here

$$
\begin{aligned}
G_{\nu}\left(\mathbf{q}, \mathbf{G}_{\|}, g_{y}^{f}-g_{y}^{i}, z\right)= & \sum_{R_{\alpha, z}} \hat{\varepsilon}_{\mathbf{q}, \nu}\left(R_{\alpha, z}\right) \\
& \times \mathbf{F}_{0}\left(q_{x}+G_{x}, q_{y}+G_{y}+g_{y}^{f}-g_{y}^{i}, z-R_{\alpha, z}\right) \\
& \times e^{-i G_{x} R_{\alpha, x}} e^{-i\left(G_{y}+g_{y}^{f}-g_{y}^{i}\right) R_{\alpha, y}},
\end{aligned}
$$

where $\alpha$ is a number of atomic layer in the slab

$$
\mathbf{F}_{0}(\mathbf{k}, z)=-i \mathbf{k} V_{s c}(\mathbf{k}, z)+\hat{z} \frac{d}{d z} V_{s c}(\mathbf{k}, z)
$$

and $\mathbf{G}_{\|}$is the 2D reciprocal lattice vector, which fulfills the relation 


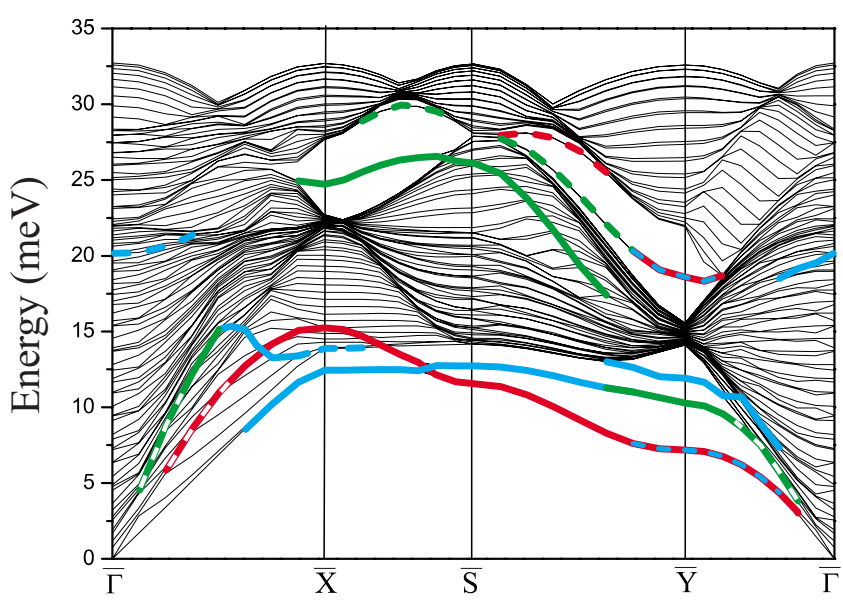

FIG. 1. (Color online) Phonon-dispersion curves for the $\mathrm{Cu}(110)$ surface. The surface modes are shown by color lines: green, red, and blue lines are $X, Y$, and $Z$ polarized modes, respectively. Solid lines correspond to vibrations localized mainly in the first layer while dashed ones denote the modes localized mostly in the second layer.

$$
\pm\left(\mathbf{k}_{f}-\mathbf{k}_{i}\right)=\mathbf{q}+\mathbf{G}_{\|}
$$

where plus and minus signs correspond to the phonon absorption and emission processes, respectively.

\section{CALCULATION RESULTS AND DISCUSSION}

The calculated phonon dispersion curves along the highsymmetry directions of the two-dimensional Brillouin zone for $\mathrm{Cu}(110)$ are shown in Fig. 1, where the surface modes and their polarization are denoted by color lines. The embedded atom interatomic potentials describe the phonon spectrum of the $\mathrm{Cu}(110)$ as well as $\mathrm{Ag}(110)$ and $\mathrm{Pd}(110)$ in good agreement with available experimental data and other theoretical results..$^{40,41}$ One can also note that the vibrational spectra of all the surfaces considered are alike.

The electronic band structure obtained by using the model pseudopotential for the $\mathrm{Cu}(110)$ surface is schematically shown in Fig. 2. Possible transitions of the excited electron in the unoccupied $\mathrm{SS}_{1}$ state or the hole in the occupied $\mathrm{SS}_{2}$ state due to scattering by phonons to the bulk electron states are illustrated by arrows.

In Fig. 3 the calculated Eliashberg function for unoccupied and occupied surfaces states on $\mathrm{Cu}(110)$ is shown together with the direction-projected local densities of phonon surfaces states and charge-density distribution of these electronic surface states. The $\alpha^{2} F(\omega)$ of the unoccupied state is mostly determined by $Z$-polarized vibrations of the first atomic layer atoms. These vibrations couple to the $\mathrm{SS}_{1}$ which is mostly localized just above the surface atoms. In particular, the main sharp peak of the Eliashberg function at 12.5 $\mathrm{meV}$ results from the strong localized surface phonon $Z$ mode, which extends over the BZ boundary below the bulk phonon spectrum (Fig. 1). The integration of the inverse frequency-weighted spectral function for this state in accordance with Eq. (2) gives $e$-ph coupling parameter $\lambda=0.08$.

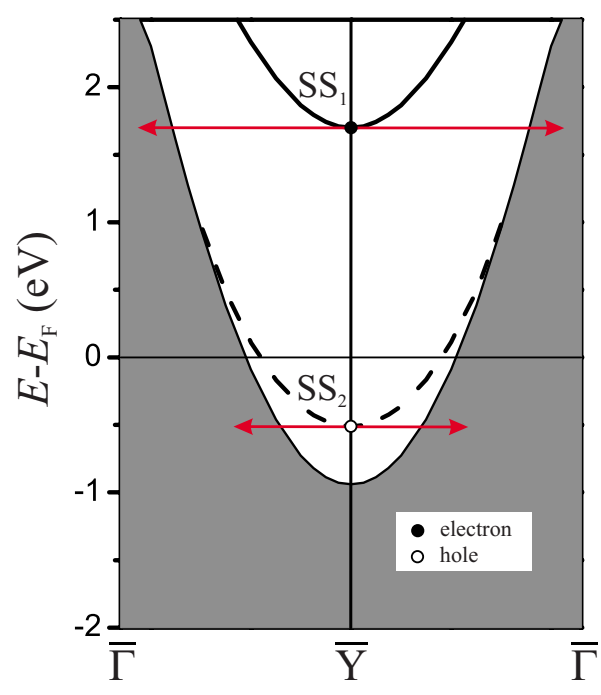

FIG. 2. (Color online) Schematic surface band structure of $\mathrm{Cu}(110)$. The white area is the $\overline{\mathrm{Y}}$ band gap supporting the SS states, the gray area represents the bulk electronic bands projected onto the $\mathrm{Cu}(110)$ surface. Solid and dashed black lines correspond to unoccupied $\left(\mathrm{SS}_{1}\right)$ and occupied $\left(\mathrm{SS}_{2}\right)$ surface states, respectively. The red arrows indicate the phonon-mediated excited electron (hole) transitions from the $\mathrm{SS}_{1}\left(\mathrm{SS}_{2}\right)$ state at $\overline{\mathrm{Y}}$ to the bulk states.

The frequency distribution of $\alpha^{2} F(\omega)$ for the $\mathrm{SS}_{1}$ state is similar to that for the $\bar{\Gamma}$ surface state on the (111) noble metal surfaces ${ }^{18,21,24}$ where vertical vibrations of the surface atoms (Rayleigh mode) provide a sharp peak and give a main contribution to the total phonon-induced decay. This similarity results from analogous charge-density distribution of the $s-p_{z}$ surface state on the (111) surface.

Contrary to the previous case, where the Eliashberg function has the sharp dominating peak, $\alpha^{2} F(\omega)$ for the occupied surface state [Fig. 3(b)] is broad and has several peaks. This leads to significantly higher $e$-ph coupling parameter, $\lambda$ $=0.24$. This value is in close agreement with $\lambda=0.23 \pm 0.02$ obtained by Straube et al. from the measured temperaturedependent photoemission data for $\mathrm{Cu}(110) .{ }^{29} \alpha^{2} F(\omega)$ of the occupied state is mainly formed by scattering processes provided by the $Y$-polarized vibrations of the first-layer atoms together with the $Z$-polarized phonon modes localized at the second-layer atoms. The first intense peak of $\alpha^{2} F(\omega)$ at $\sim 7 \mathrm{meV}$ results from the mixed $\left(Y_{1}\right.$ and $\left.Z_{2}\right)$ vibration mode localized in the vicinity of the $\bar{Y}$ point (see Fig. 1). The next higher frequency peak of Eliashberg function at $\sim 11 \mathrm{meV}$ is predominantly determined by the $Y$-polarized vibrational mode (located below the bulk phonons) localized at the first atomic plane, mainly by a flat part of this band in the vicinity of the $\overline{\mathrm{S}}$ symmetry point of the BZ. The peak at $15.8 \mathrm{meV}$ as well as the peak at $\sim 20 \mathrm{meV}$ results mostly from scattering by the $Z$-polarized phonon resonances localized at the second layer at the $\bar{X}$ point at the bottom of bulk phonon spectrum and at the $\overline{\Gamma X}$ direction, respectively. The structure at $\sim 20 \mathrm{meV}$ has also contribution from scattering by the $Y$-polarized surface phonon mode localized in the $\overline{\mathrm{Y}}$ gap. The different mechanism of the phonon-mediated scattering for excited hole in the occupied surface state with respect to 
(a)

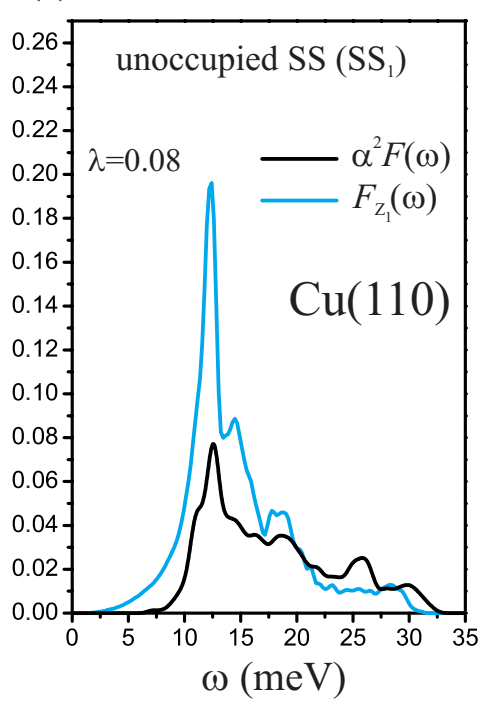

(b)

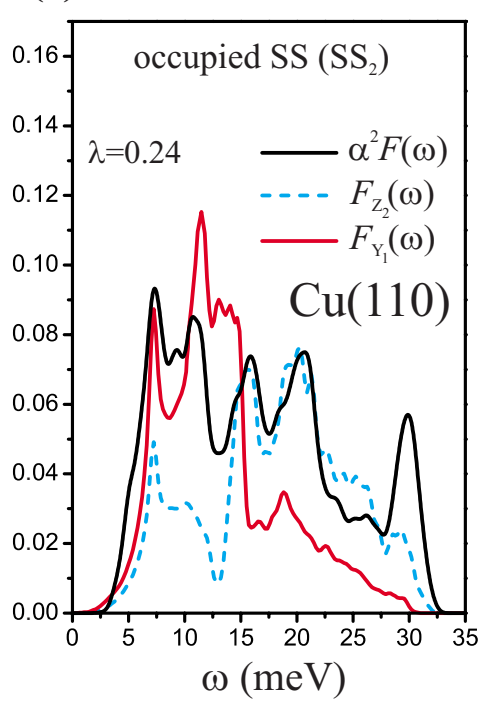

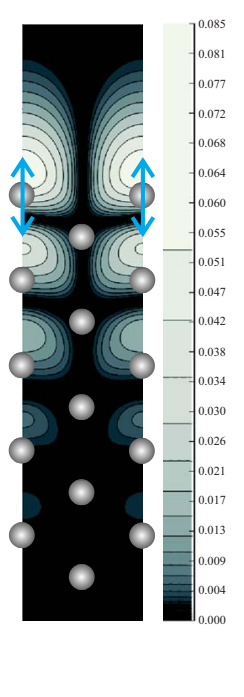

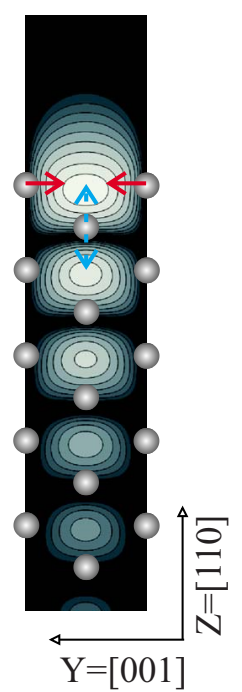

FIG. 3. (Color online) Electron-phonon Eliashberg spectral function $\alpha^{2} F(\omega)$ for the $\overline{\mathrm{Y}}$ (a) unoccupied and (b) occupied (black lines) surface states on $\mathrm{Cu}(110)$. The $Y$ (red line) and the $Z$ (blue line) direction projected surface phonon densities of states $F(\omega)$ for the first- (solid line) and the second- (dashed line) surface layers are shown. Charge-density distribution for the (a) unoccupied and (b) occupied surface states are given in a logarithmic scale. Grey balls denote projections of atoms of a real crystalline fcc structure onto the (110) plane. Red and blue arrows show $Y$ and $Z$ polarization vectors of the surface phonons which mostly contribute to the $e$-ph scattering.

that for electrons in the unoccupied state results from different spatial localization of these states. The maximum of the $\mathrm{SS}_{2}$ charge density is localized just above the second-layer atoms while the maximum of the $\mathrm{SS}_{1}$ charge density is situated above the first-layer atoms. Thus, these states are related to different atomic chains of the (110) surface and maxima of charge density of the occupied state lie at minima of the unoccupied state. As a result of this, the distinct vibrational

TABLE I. Calculated electron-phonon coupling parameter.

\begin{tabular}{lccc}
\hline \hline & $\mathrm{Cu}(110)$ & $\operatorname{Ag}(110)$ & $\operatorname{Pd}(110)$ \\
\hline $\mathrm{SS}_{1}$ & 0.08 & 0.08 & 0.03 \\
$\mathrm{SS}_{2}$ & 0.24 & 0.28 & 0.17 \\
\hline \hline
\end{tabular}

displacements contribute to the quasiparticle decay in these states.

The scattering picture for the occupied SS is rather similar to that in $1 \mathrm{ML} \mathrm{Na/Cu}(111)$ quantum well $(\mathrm{QW}){ }^{36,42}$ In the latter system the scattering of excited holes localized in the sodium QW is mainly due to coupling with the in-plane vibrations of $\mathrm{Na}$ atoms and with the $Z$-polarized vibrations of the top $\mathrm{Cu}$ substrate layer atoms.

The similar values for the $e$ - $p h$ coupling parameters were obtained for occupied and unoccupied surface states on the $\operatorname{Ag}(110)$ surface (see Table I). It should be noted that the simple average of the calculated values of $\lambda$ for both $\bar{Y}$ surface states on $\operatorname{Ag}(110)$ agrees well with $\lambda=0.13 \pm 0.02,{ }^{28}$ which includes contributions both from occupied and unoccupied surface states. Despite the unoccupied character of the lower SS on $\operatorname{Pd}(110)$ the general trend, namely, a small $\lambda_{\mathrm{SS}_{1}}$ and significantly bigger $\lambda_{\mathrm{SS}_{2}}$, is also obtained for this surface. However, both $\lambda_{\mathrm{SS}_{1}}$ and $\lambda_{\mathrm{SS}_{2}}$ are smaller than the respective $e-p h$ coupling parameters on $\mathrm{Cu}(110)$ and $\operatorname{Ag}(110)$. This can be explained by smaller density of bulk electronic states involved in the phonon-mediated SS electron scattering on $\mathrm{Pd}(110)$. This tendency is in accordance with the density-functional perturbation theory calculation result for electron-phonon coupling parameter in the $\bar{\Gamma}$ unoccupied surface state on $\operatorname{Pd}(111),{ }^{27}$ which was obtained to be 0.08 , significantly smaller compared to $\lambda$ in the respective occupied SS on $\mathrm{Cu}(111)$ and $\mathrm{Ag}(111){ }^{21,27}$

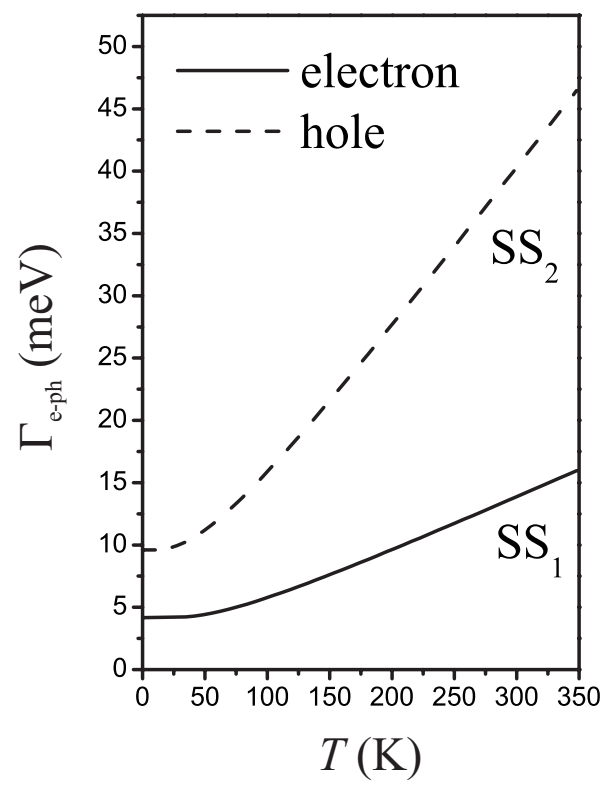

FIG. 4. Temperature dependence of the phonon-induced lifetime broadening, $\Gamma_{e-p h}$, for the unoccupied (solid line) and occupied (dashed line) surface states at the $\overline{\mathrm{Y}}$ symmetry point on $\mathrm{Cu}(110)$. 
Figure 4 shows the calculated linewidth of unoccupied and occupied surface states on $\mathrm{Cu}(110)$ as a function of temperature. At zero-temperature limit, $\Gamma_{e-p h}=4.2 \mathrm{meV}$ obtained for the excited electron is significantly smaller than $\Gamma_{e-p h}$ $=9.6 \mathrm{meV}$ calculated for the excited hole. For temperatures above $100 \mathrm{~K}$ the computed temperature dependence can be described by using high-temperature asymptotic behavior $\Gamma_{e-p h}=2 \pi \lambda k_{\mathrm{B}} T$, which can directly be obtained from measurements of temperature dependence of the linewidths of the surface states. At RT the difference between hole and electron $\Gamma_{e-p h}$ amounts to $26 \mathrm{meV}$. This value is comparable with the electron-defect scattering contribution to the linewidth (30 meV) obtained for Au(111) at RT (Ref. 18) and is significantly higher than the electron-electron contribution in the noble metal surface states. ${ }^{8}$

\section{CONCLUSION}

We have presented results of comparative study of the electron-phonon scattering of excited holes and electrons in occupied and unoccupied $\overline{\mathrm{Y}}$ surface states on $\mathrm{Cu}(110)$, $\operatorname{Ag}(110)$, and $\operatorname{Pd}(110)$. We show that in the unoccupied surface state on $\mathrm{Cu}(110)$ and $\mathrm{Ag}(110)$ the e-ph coupling param- eter $\lambda$ is about three times smaller than that in the occupied surface state due to distinct phonon modes that contribute to $e-p h$ scattering. The calculated $e$ - $p h$ coupling parameter for the occupied SS on the $\mathrm{Cu}(110)$ agrees well with the value obtained from the temperature-dependent photoemission measurements. ${ }^{29}$ The difference in mechanisms of the phonon-mediated scattering for excited holes and electrons results from distinct spatial localization of occupied and unoccupied states. As a result, the different vibrational displacements contribute to the quasiparticle decay in these states. In spite of both $\bar{Y}$ surface states on Pd(110) are unoccupied they have spatial distribution similar to other considered noble metal surfaces that leads to different rates of the phonon-mediated decay in these states.

\section{ACKNOWLEDGMENTS}

We gratefully acknowledge partial support by SB RAS (Project 3.6.1.1.), the Department of Education of the Basque Country Governement, the University of the Basque Country (project GV-UPV/EHU, Grant No. IT-366-07), and Ministerio de Ciencia y Tecnología (grant FIS2007-66711-C02-01). The calculations were performed on the SKIF-Cyberia supercomputer of the Tomsk State University. *waptctce@sq.ehu.es

${ }^{1}$ G. Borstel and G. Thörner, Surf. Sci. Rep. 8, 1 (1988).

${ }^{2}$ E. V. Chulkov, V. M. Silkin, and E. N. Shirykalov, Surf. Sci. 188, 287 (1987).

${ }^{3}$ H. L. Davis, J. B. Hannon, K. B. Ray, and E. W. Plummer, Phys. Rev. Lett. 68, 2632 (1992).

${ }^{4}$ V. Chis and B. Hellsing, Phys. Rev. Lett. 93, 226103 (2004).

${ }^{5}$ L. I. Johansson, H. I. P. Johansson, J. N. Andersen, E. Lundgren, and R. Nyholm, Phys. Rev. Lett. 71, 2453 (1993).

${ }^{6}$ P. J. Feibelman and R. Stumpf, Phys. Rev. B 50, 17480 (1994).

${ }^{7}$ J. Osma, I. Sarría, E. V. Chulkov, J. M. Pitarke, and P. M. Echenique, Phys. Rev. B 59, 10591 (1999).

${ }^{8}$ E. V. Chulkov, V. M. Silkin, and M. Machado, Surf. Sci. 482485, 693 (2001).

${ }^{9}$ N. Memmel and E. Bertel, Phys. Rev. Lett. 75, 485 (1995).

${ }^{10}$ M. Giesen, G. Schulze Icking-Konert, and H. Ibach, Phys. Rev. Lett. 82, 3101 (1999).

${ }^{11}$ Photochemistry and Photophysics on Surfaces, edited by J. T. Yates, Jr. and H. Petek, special issue of Chem. Rev. 106(10), (2006).

${ }^{12}$ H. Nienhaus, Surf. Sci. Rep. 45, 1 (2002).

${ }^{13}$ G. A. Somorjai, in Elementary Reaction Step in Heterogeneous Catalysis, edited by R. W. Joyner and R. A. Van Santen (Kluwer, Dordrecht, 1993), p. 3.

${ }^{14}$ J. Massardier, J. C. Bertolini, and A. Renouprez, Proceedings of the Ninth International Conference on Catalysis, Calgary, 1988, Vol. 3, p. 1222.

${ }^{15}$ B. A. McDougall, T. Balasubramanian, and E. Jensen, Phys. Rev. B 51, 13891 (1995).

${ }^{16}$ J. E. Gayone, S. V. Hoffmann, Z. Li, and Ph. Hofmann, Phys. Rev. Lett. 91, 127601 (2003).
${ }^{17}$ M. Fuglsang Jensen, T. K. Kim, S. Bengió, I. Y. Sklyadneva, A. Leonardo, S. V. Eremeev, E. V. Chulkov, and Ph. Hofmann, Phys. Rev. B 75, 153404 (2007).

${ }^{18}$ S. V. Eremeev and E. V. Chulkov, Phys. Solid State 51, 854 (2009).

${ }^{19}$ E. Knoesel, A. Hotzel, and M. Wolf, J. Electron Spectrosc. Relat. Phenom. 88-91, 577 (1998).

${ }^{20}$ S. LaShell, E. Jensen, and T. Balasubramanian, Phys. Rev. B 61, 2371 (2000).

${ }^{21}$ A. Eiguren, B. Hellsing, F. Reinert, G. Nicolay, E. V. Chulkov, V. M. Silkin, S. Hüfner, and P. M. Echenique, Phys. Rev. Lett. 88, 066805 (2002).

${ }^{22}$ S. J. Tang, Ismail, P. T. Sprunger, and E. W. Plummer, Phys. Rev. B 65, 235428 (2002).

${ }^{23}$ R. Matzdorf, Surf. Sci. Rep. 30, 153 (1998).

${ }^{24}$ A. Eiguren, B. Hellsing, E. V. Chulkov, and P. M. Echenique, Phys. Rev. B 67, 235423 (2003).

${ }^{25}$ T. K. Kim, T. S. Sorensen, E. Wolfring, H. Li, E. V. Chulkov, and Ph. Hofmann, Phys. Rev. B 72, 075422 (2005).

${ }^{26}$ J. Kröger, Rep. Prog. Phys. 69, 899 (2006).

${ }^{27}$ I. Yu. Sklyadneva, R. Heid, V. M. Silkin, A. Melzer, K.-P. Bohnen, P. M. Echenique, Th. Fauster, and E. V. Chulkov, Phys. Rev. B 80, 045429 (2009).

${ }^{28}$ S. M. Dounce, M. Yang, and H.-L. Dai, Phys. Rev. B 67, 205410 (2003).

${ }^{29}$ P. Straube, F. Pforte, T. Michalke, K. Berge, A. Gerlach, and A. Goldmann, Phys. Rev. B 61, 14072 (2000).

${ }^{30}$ S. S. Tsirkin, S. V. Eremeev, and E. V. Chulkov, Phys. Solid State 52, 188 (2010).

${ }^{31}$ S. S. Tsirkin, S. V. Eremeev, and E. V. Chulkov, Surf. Sci. 604, 804 (2010). 
${ }^{32}$ O. Zeybek, A. M. Davarpanah, and S. D. Barrett, Surf. Sci. 600, 5176 (2006).

${ }^{33}$ A. Gerlach, G. Meister, R. Matzdorf, and A. Goldmann, Surf. Sci. 443, 221 (1999).

${ }^{34}$ A. Goldmann, V. Dose, and G. Borstel, Phys. Rev. B 32, 1971 (1985).

${ }^{35} \mathrm{G}$. Grimvall, The Electron-Phonon-Interaction in Metals (NorthHolland, Amsterdam, 1981).

${ }^{36}$ S. V. Eremeev, I. Yu. Sklyadneva, P. M. Echenique, S. D. Borisova, G. Benedek, G. G. Rusina, and E. V. Chulkov, Surf. Sci. 601, 4553 (2007).
${ }^{37}$ S. Mathias, S. V. Eremeev, E. V. Chulkov, M. Aeschlimann, and M. Bauer, Phys. Rev. Lett. 103, 026802 (2009).

${ }^{38}$ N. W. Ashcroft, Phys. Lett. 23, 48 (1966).

${ }^{39}$ E. V. Chulkov, V. M. Silkin, and P. M. Echenique, Surf. Sci. 437, 330 (1999).

${ }^{40}$ R. Heid and K.-P. Bohnen, Phys. Rep. 387, 151 (2003).

${ }^{41}$ A. V. Bertsch, E. V. Chulkov, S. V. Eremeev, A. G. Lipnitskii, G. G. Rusina, and I. Yu. Sklyadneva, Vacuum 46, 625 (1995).

${ }^{42}$ E. V. Chulkov, J. Kliewer, R. Berndt, V. M. Silkin, B. Hellsing, S. Crampin, and P. M. Echenique, Phys. Rev. B 68, 195422 (2003). 